\title{
Immunohistochemical testing of conventional adenomas for loss of expression of mismatch repair proteins in Lynch syndrome mutation carriers: a case series from the Australasian site of the colon cancer family registry
}

Michael D Walsh ${ }^{1,2}$, Daniel D Buchanan ${ }^{1}$, Sally-Ann Pearson ${ }^{1}$, Mark Clendenning ${ }^{1}$, Mark A Jenkins ${ }^{3}$, Aung Ko Win ${ }^{3}$, Rhiannon J Walters ${ }^{1}$, Kevin J Spring ${ }^{1}$, Belinda Nagler ${ }^{1}$, Erika Pavluk $^{1}$, Sven T Arnold ${ }^{1}$, Jack Goldblatt ${ }^{4,5}$, Jill George ${ }^{5}$, Graeme K Suthers ${ }^{6,7}$, Kerry Phillips ${ }^{7}$, John L Hopper ${ }^{3}$, Jeremy R Jass ${ }^{8}$, John A Baron ${ }^{9}$, Dennis J Ahnen ${ }^{10}$, Stephen N Thibodeau ${ }^{11}$, Noralane Lindor ${ }^{12}$, Susan Parry ${ }^{13}$, Neal I Walker ${ }^{14}$, Christophe Rosty ${ }^{1,2,15}$ and Joanne P Young ${ }^{1,2}$

${ }^{1}$ Familial Cancer Laboratory, QIMR, Herston, QLD, Australia; ${ }^{2}$ School of Medicine, University of Queensland, Herston, QLD, Australia; ${ }^{3}$ Centre for MEGA, School of Population Health, University of Melbourne, Carlton, VIC, Australia; ${ }^{4}$ School of Paediatrics and Child Health, University of Western Australia, Nedlands, WA, Australia; ${ }^{5}$ Genetic Services of Western Australia, Subiaco, WA, Australia; ${ }^{6}$ Department of Paediatrics, University of Adelaide, Adelaide, SA, Australia; ${ }^{7}$ South Australian Clinical Genetics Service, North Adelaide, SA, Australia; ${ }^{8}$ Department of Cellular Pathology, St Mark's Hospital, Harrow, UK; ${ }^{9}$ Department of Medicine, University of North Carolina, Chapel Hill, NC, USA; ${ }^{10}$ Denver Department of Veterans Affairs Medical Center and University of Colorado School of Medicine, Denver, CO, USA;

${ }^{11}$ Division of Laboratory Genetics, Mayo Clinic, Rochester, MN, USA; ${ }^{12}$ Departments of Laboratory Medicine and Pathology and Medical Genetics, Mayo Clinic, Rochester, Minnesota, USA; ${ }^{13}$ New Zealand Familial Gastrointestinal Cancer Registry, Auckland City Hospital, Auckland, New Zealand; ${ }^{14}$ Envoi Pathology, Herston, QLD, Australia and ${ }^{15}$ Pathology Queensland, Royal Brisbane and Women's Hospital, Herston, $Q L D$, Australia

\begin{abstract}
Debate continues as to the usefulness of assessing adenomas for loss of mismatch repair protein expression to identify individuals with suspected Lynch syndrome. We tested 109 polyps from 69 proven mutation carriers (35 females and 34 males) belonging to 49 Lynch syndrome families. All polyps were tested by immunohistochemistry for four mismatch repair proteins MLH1, MSH2, MSH6 and PMS2. Detailed pathology review was performed by specialist gastrointestinal pathologists. The majority of polyps (86\%) were conventional adenomas $(n=94)$, with 65 tubular and 28 tubulovillous adenomas and a single villous adenoma. The remaining 15 lesions (14\%) were serrated polyps. Overall, loss of mismatch repair expression was noted for 78/ $109(72 \%)$ of polyps. Loss of mismatch repair expression was seen in 74 of $94(79 \%)$ conventional adenomas, and 4 of $15(27 \%)$ serrated polyps from mismatch repair gene mutation carriers. In all instances, loss of expression was consistent with the underlying germline mutation. Mismatch repair protein expression was lost in 27 of 29 adenomas with a villous component compared with 47 of 65 adenomas without this feature $(93$ vs $73 \% ; P=0.028$ ). A strong trend was observed for high-grade dysplasia. Mismatch repair deficiency was observed in 12 of 12 conventional adenomas with high-grade dysplasia compared with 60 of 79 with low-grade
\end{abstract}

Correspondence: Dr JP Young, Familial Cancer Laboratory, Queensland Institute of Medical Research, Herston QLD 4006, Australia. E-mail: Joanne.Young@qimr.edu.au

Received 6 September 2011; revised 26 October 2011; accepted 9 November 2011; published online 10 February 2012 
dysplasia (100 vs $76 \% ; P=0.065$ ). We were unable to demonstrate a significant association between conventional adenoma size or site and mismatch repair deficiency. All (4/4 or $100 \%)$ of the serrated polyps demonstrating mismatch repair deficiency were traditional serrated adenomas from a single family. Diagnostic testing of adenomas in suspected Lynch syndrome families is a useful alternative in cases where cancers are unavailable. The overwhelming majority of conventional adenomas from mutation carriers show loss of mismatch repair protein expression concordant with the underlying germline mutation.

Modern Pathology (2012) 25, 722-730; doi:10.1038/modpathol.2011.209; published online 10 February 2012

Keywords: adenomas; Lynch syndrome

Lynch syndrome is an inherited disorder of deficient DNA mismatch repair, which predisposes to a high risk of young-onset colorectal cancer as well as cancers at extra-colonic sites. Colorectal cancers in Lynch syndrome are thought to develop via the common adenoma-carcinoma developmental pathway with few exceptions. ${ }^{1-3}$ The risk of malignant transformation in conventional adenomas in general has traditionally been associated with features, such as size (larger adenomas are more likely to undergo transformation), dysplasia (risk of transformation varies directly with the grade of dysplasia), and the presence of advanced features (adenomas with villous components are considered to be of higher risk than adenomas without this morphological feature). ${ }^{4}$ Consistent with a high risk of malignant transformation, conventional adenomas arising in Lynch syndrome have also been reported to more frequently demonstrate villous components and highgrade dysplasia, and are thought to be larger than those in the general population. ${ }^{5}$ In addition, further reports have suggested that adenomas from mismatch repair mutation carriers include flat appearance and rapid growth. ${ }^{6}$ Adenomas are relatively uncommon before the age of 50 years, in both Lynch syndrome mutation carriers ${ }^{1,5}$ and in the general population. ${ }^{7}$ Although Lynch syndrome patients are thought to develop adenomas at a similar rate as, and in a similar spontaneous manner to, the general population, Lynch syndrome adenomas once established, are more likely to undergo malignant conversion and to be located in the proximal colon. ${ }^{5,6}$ It is likely that micro-adenomas in Lynch syndrome mutation carriers do not remain dormant for many years as is likely to be the case for the general population. ${ }^{5}$ The demonstration of mismatch repair deficiency in adenomas from mutation carriers points to establishment in an early premalignant phase during colorectal cancer development.

Lynch syndrome can be difficult to diagnose on clinical criteria because there are no phenotypic signs in the individual such as polyposis. ${ }^{8}$ In addition, family history is not always available, nor is it always of a configuration that would alert the clinical team to Lynch syndrome. Now screening for Lynch syndrome can be achieved through immunostaining for mismatch repair proteins in a spectrum of Lynch syndrome-associated cancers including those of the colorectum, but it remains unclear if screening of colonic adenomas is of value for the idenitification of patients with Lynch syndrome. Screening of early-onset adenomas for mismatch repair deficiency in order to diagnose Lynch syndrome in the population has yielded disappointing results, ${ }^{9}$ suggesting that more focused testing should be evaluated. Not all Lynch syndrome adenomas found in mismatch repair mutation carriers show loss of immunostaining, and there is considerable variation in the literature about the frequency of loss of mismatch repair protein expression in this setting. ${ }^{8,10-13}$ In this large case series of 109 polyps from 69 proven mismatch repair mutation carriers, we demonstrate that the range of adenoma types in which mismatch repair expression loss can be demonstrated is considerably more diverse than has been previously thought.

\section{Materials and methods}

Patients described in this report were enrolled in the Australasian Colorectal Cancer Family Study. ${ }^{14}$ They had institutional review board approval under the policies and procedures of the Colon Cancer Family Registry for recruitment of participants and protocols for carrying out research projects. Germline mutation in MLH1, MSH2, MSH6 or PMS2 had been determined as previously reported. ${ }^{15}$ Patients were selected on the basis of being a proven mismatch repair gene mutation carrier and having undergone a polypectomy from which tissue blocks were available. All polyps underwent a standard review by one specialist gastrointestinal pathologist (JRJ, NIW or CR) blinded to the mismatch repair immunohistochemistry results of each polyp. Polyps were assessed for histological sub-type, reported size (where available from endoscopy report), site in the colorectum (where known), villous component and grade of dysplasia. All polyps had been tested by immunohistochemistry (immunohistochemistry) for the four mismatch repair proteins MLH1, MSH2, MSH6 and PMS2 as previously described ${ }^{16}$ using a Dako Cytomation automated staining machine, and visual assessment. Polyps that demonstrated protein loss were designated mismatch repair deficient. In addition, microsatellite instability testing using a panel of 10 markers (incorporating the standard National Cancer Institute panel) was also carried out 
as described in a previous report ${ }^{16}$ on 66 conventional adenomas and 14 serrated polyps. MLH1 methylation testing was performed, as reported previously, ${ }^{2,16}$ on four serrated polyps with MLH1 immunodeficiency to determine whether loss of mismatch repair in these lesions was due to a somatic methylation event. ${ }^{17}$ Somatic BRAF c.1799T $>$ A (p.V600E) mutation testing was undertaken on all serrated polyps as described in previous reports ${ }^{2,16}$ to determine whether serrated lesions in mismatch repair mutation carriers develop via the same pathway observed in the general population. Statistical analysis was carried out using Statistical Package for Social Sciences (SPSS version 19.0 for Macintosh). Contingency tables were assessed using Fisher's exact test. Differences between means were assessed using a Student's $t$-test. To test for equality of the variance between groups probability plots and an F-test were used. All reported statistical tests were two-sided and $P$-values of $<0.05$ were considered statistically significant.

\section{Results}

\section{Polyps}

We examined 109 polyps from 69 proven mutation carriers (35 females and 34 males) arising in 49 Lynch syndrome families (14 carried a germline mutation in MLH1, 26 in MSH2, 6 in MSH6 and 3 in PMS2). Of 109 polyps, 94 were conventional adenomas comprising 65 tubular, 28 tubulovillous and 1 villous adenoma. Of the conventional adenomas, 6/64 (10\%) tubular adenomas demonstrated high-grade dysplasia compared with 6/26 (23\%) tubulovillous adenomas $(P=0.098)$. The average age at polypectomy for each polyp studied was $49 \pm 12$ SD years (ranging from 22 to 89 years). The average age at polypectomy for tubulovillous adenomas ( $47 \pm 15$ yrs SD) was significantly younger than the age at polypectomy for tubular adenomas (53 \pm 11 yrs SD) $(P=0.032)$. The remaining 15 polyps were serrated polyps comprising 5 microvesicular hyperplastic polyps, 5 sessile serrated adenomas/polyps and 5 traditional serrated adenomas. ${ }^{18}$ The average age at which serrated polyps were removed was $42 \pm 7$ years SD, significantly younger than the average age at removal of conventional adenomas at $51 \pm 12.4$ yrs $\operatorname{SD}(P=0.006)$. A summary of polyp characteristics is shown in Table 1.

\section{Mismatch Repair Expression Loss}

Overall, loss of mismatch repair expression was observed in 78/109 polyps (72\%). In all, 74 of 94 confirmed conventional adenomas $(79 \%)$ from mismatch repair mutation carriers showed loss of expression of mismatch repair proteins. An example of expression loss in a low-grade ademoma is shown in Figure 1. These included 34/37 (92\%) from MLH1

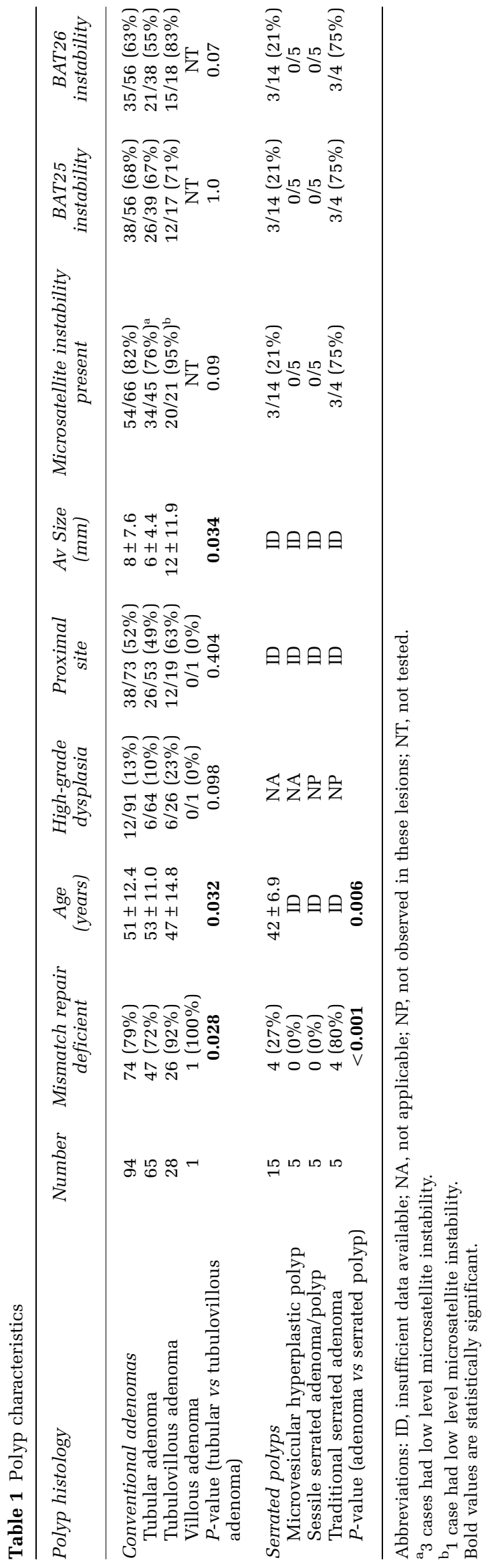



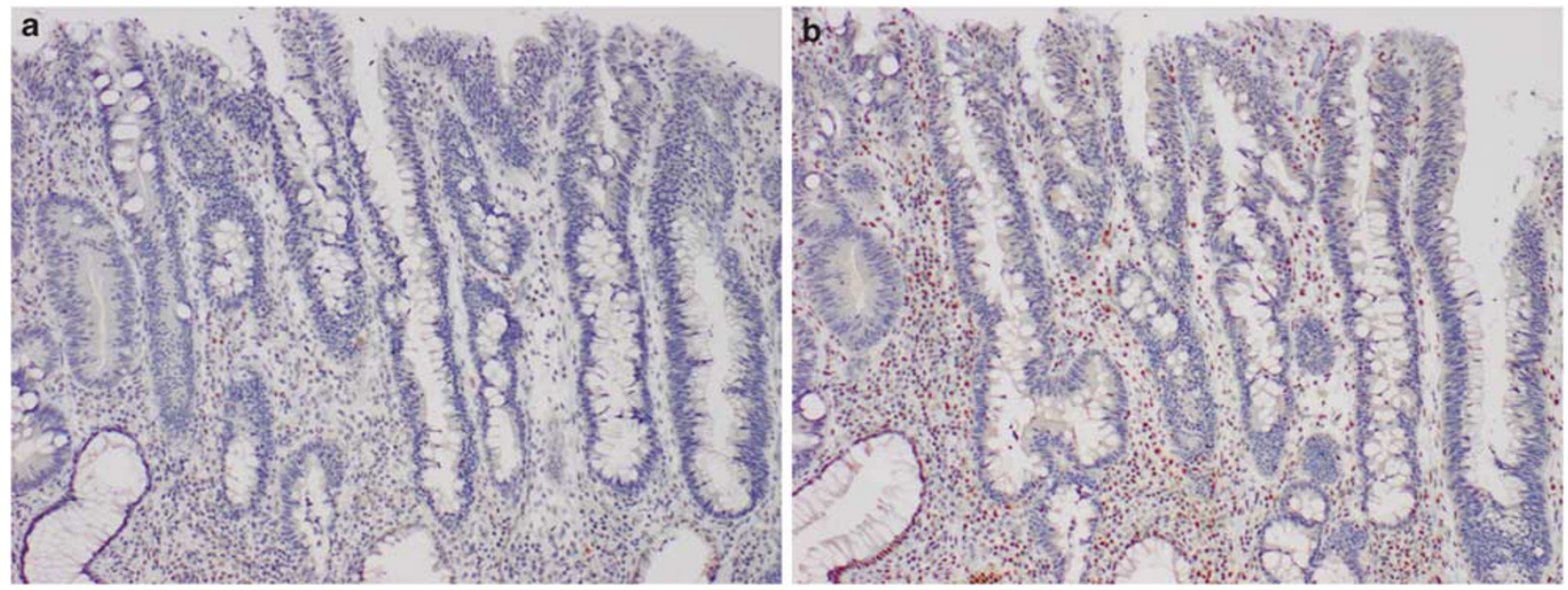

Figure 1 Example of loss of expression of mismatch repair proteins in a tubular adenoma with low-grade dysplasia from a patient with germline mutation in MLH1. Figure shows loss of MLH1 (a) and PMS2 (b) in adenoma cells.

mutation carriers, 34/44 (77\%) from $M S H 2,4 / 11$ $(36 \%)$ from MSH6, and $2 / 2(100 \%)$ from PMS2 mutation carriers. No statistical difference in patient ages at the time of polypectomy between mismatch repair deficient and proficient adenomas was observed ( $49 \pm 12$ yrs SD vs $50 \pm 11$ yrs SD; $P=0.82$ ). High-level microsatellite instability results were concordant with mismatch repair protein loss results in 63/66 (95.6\%) evaluable conventional adenomas and 14/14 (100\%) evaluable serrated polyps. Microsatellite instability was detected more frequently in tubulovillous (95\%) than in tubular $(76 \%)$ adenomas but the difference failed to reach statistical significance $(P=0.09)$. There was no significant difference between the detection rate of markers BAT26 and BAT25 $(P=0.69)$. In all instances where mismatch repair protein was absent, loss of expression was consistent with the underlying germline mutation, and was continuously absent or occasionally reduced in the lesion. Reduced pattern expression loss of MLH1 was observed in four adenomas. Three arose in a single 68-year-old male mutation carrier with an MLH1-splicing mutation (c.790+2dupT r. (678_790del, 678_884del) p.?). A second patient, a 43-year-old male, also a splicing mutation carrier in MLH1 (c.1559-2A > T r.spl? p.?) showed reduced intensity of immunolabelling for MLH1 and complete loss of PMS2 expression (Figure 2).

\section{Site and Size}

Site was able to be determined for 73 conventional adenomas (38 proximal and 35 distal). Adenomas overall were significantly smaller in the distal colorectum compared with those derived from the proximal colon (average size $4.89 \mathrm{~mm}$ vs $8.11 \mathrm{~mm}$; $P=0.004)$. When site was considered, there was no difference between the prevalence of mismatch repair deficient adenomas in the proximal colon $(31 / 38)$ and the distal colorectum (30/35) (82 vs $86 \% ; P=0.76$ ). Similarly, size did not appear to have a major role in determining whether an adenoma would demonstrate loss of mismatch repair expression. In all, 39 of 55 adenomas (71\%) less than $10 \mathrm{~mm}$ in size demonstrated loss of mismatch repair protein compared with 13 of $17(76 \%)$ of polyps $10 \mathrm{~mm}$ or greater in size $(P=0.76)$. Further, 28 of 35 $(80 \%)$ adenomas less than $5 \mathrm{~mm}$ in size also showed loss of mismatch repair proteins. The smallest mismatch repair deficient adenomas were $2 \mathrm{~mm}$ in size $(n=6)$.

\section{High-grade Dysplasia and Villous Component}

Mismatch repair protein expression was lost in 27 of 29 adenomas with a villous component compared with 47 of 65 adenomas without this feature (93 vs $73 \% ; P=0.028$ ). Adenomas with high-grade dysplasia were more likely to demonstrate loss of mismatch repair protein, with every high-grade adenoma demonstrating mismatch repair deficiency $(12 / 12)$ compared with 60/79 low grade adenomas (100 vs $76 \%)$, however the results did not attain statistical significance $(P=0.065)$. Adenomas with high-grade dysplasia and those with a villous component were equally likely to arise from either side of the colon. Adenomas with villous component were significantly larger than those with out this feature $(6 \pm 4.4 \mathrm{~mm}$ s.d. vs $12 \pm 11.9 \mathrm{~mm}$ s.d.; $P=0.034$ ). Detailed results for conventional adenomas are shown in Table 2.

\section{Serrated Lesions}

Fifteen serrated polyps arising in mismatch repair mutation carriers were examined for mismatch repair deficiency, and four $(27 \%)$ showed mutation-appropriate 


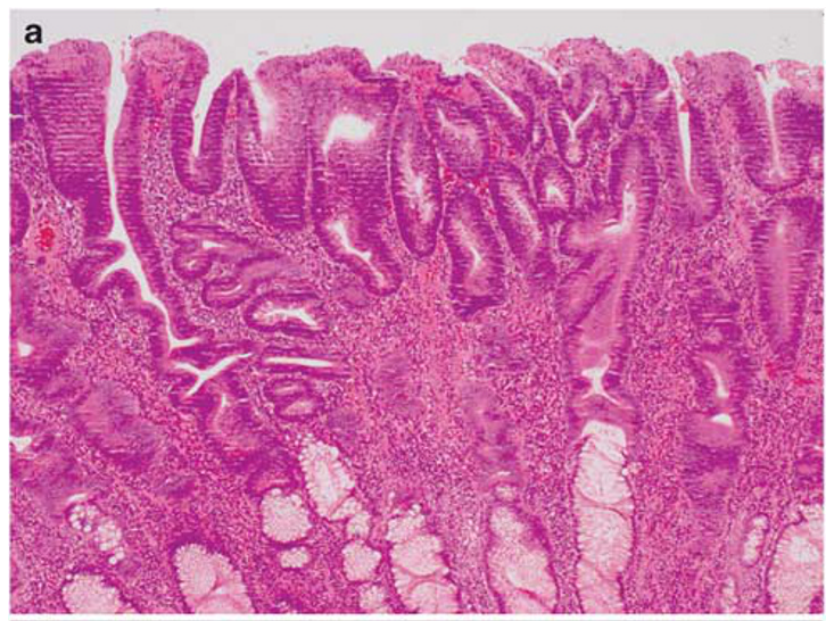

Table 2 Features of conventional adenomas tested for MMR deficiency by IHC $(n=94)$

\begin{tabular}{|c|c|c|c|c|}
\hline & $\begin{array}{c}M M R \\
\text { deficient }\end{array}$ & $\begin{array}{c}M M R \\
\text { proficient }\end{array}$ & $\mathrm{P}$-value & OR $(95 \% C I)$ \\
\hline \multicolumn{5}{|l|}{ Side } \\
\hline Left & $30(86 \%)$ & $5(14 \%)$ & 0.76 & $0.74(0.21-2.58)$ \\
\hline Right & $31(82 \%)$ & $7(18 \%)$ & & \\
\hline \multicolumn{5}{|l|}{ Size } \\
\hline$<10 \mathrm{~mm}$ & $39(71 \%)$ & $16(29 \%)$ & 0.76 & $1.33(0.38-4.71)$ \\
\hline$>=10 \mathrm{~mm}$ & $13(76 \%)$ & $4(24 \%)$ & & \\
\hline \multicolumn{5}{|l|}{ Dysplasia } \\
\hline HGD & $12(100 \%)$ & $0(0 \%)$ & 0.065 & a \\
\hline LGD & $60(76 \%)$ & $19(24 \%)$ & & \\
\hline \multicolumn{5}{|c|}{ Villous component } \\
\hline No & $47(72 \%)$ & $18(28 \%)$ & 0.028 & $5.17(1.11-24.01)$ \\
\hline Yes & $27(93 \%)$ & $2(7 \%)$ & & \\
\hline
\end{tabular}

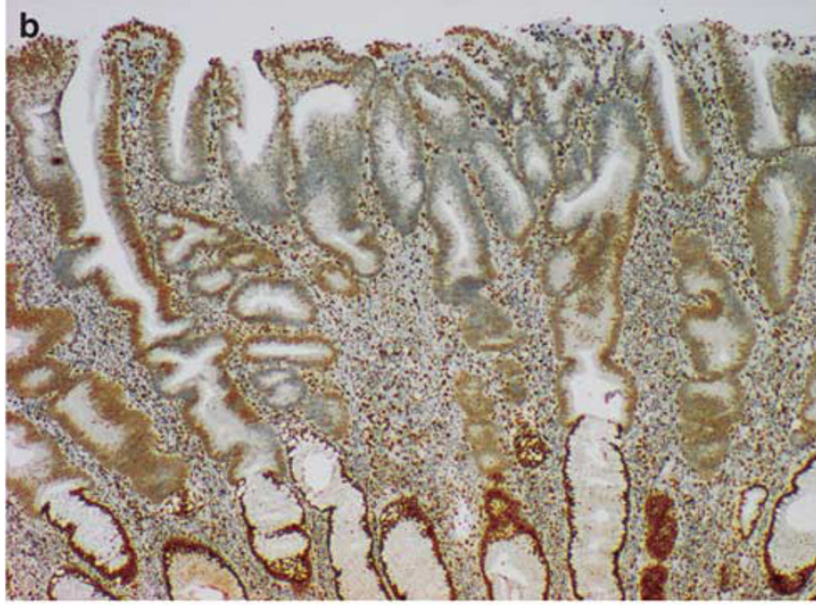

aOR not calculated on "0" field value.

Bold values are statistically significant.

mutation carriers from a single family. The family which has been previously reported elsewhere, had a serrated neoplasia predisposition segregating independently of the MLH1 mutation. ${ }^{2}$ None of the three MLH1-deficient serrated polyps demonstrated MLH1 methylation or somatic BRAF c.1799T >A (p.V600E) mutation. DNA from a fourth lesion failed to amplify after multiple attempts. Somatic BRAF c.1799T > A (p.V600E) mutation was observed in 3/5 microvesicular hyperplastic polyps (60\%), 3/4 evaluable sessile serrated adenomas/polyps (75\%), and $0 / 3$ traditional serrated adenomas.

\section{Discussion}

We examined the value of mismatch repair deficiency as a screening test for Lynch syndrome in a large series of conventional adenomas and a smaller number of serrated polyps derived from proven mismatch repair mutation carriers, and found that a diverse range of lesions may be useful for this purpose. Though the majority of previous studies find evidence of mismatch repair deficiency in at least some adenomas in mismatch repair mutation carriers, ${ }^{12,19-22}$ some studies have suggested that it is only worthwhile testing large, proximal adenomas exhibiting high-grade dysplasia, ${ }^{10}$ whereas others report a greater proportion of mismatch repair deficient adenomas from among smaller and distal lesions. ${ }^{1,8}$ In our series, the overwhelming majority of conventional adenomas in mutation carriers $(79 \%)$ showed evidence of mismatch repair deficiency. In common with previous reports,,${ }^{1,8}$ we found mismatch repair deficiency convincingly demonstrated in small lesions (as small as $2 \mathrm{~mm}$ ), ${ }^{1}$ as well as adenomas from the distal colorectum. ${ }^{8}$ There is general agreement on the direct relationship between 


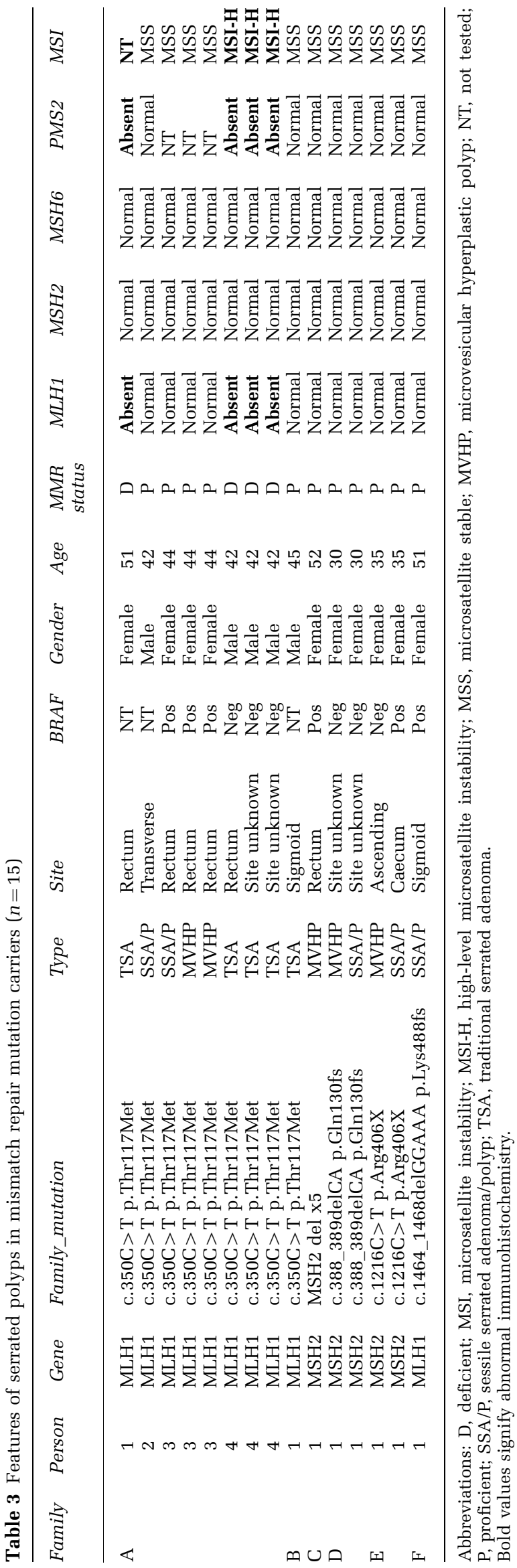

high-grade dysplasia and evidence of mismatch repair deficiency, ${ }^{1,6,8,19}$ but the size of a mismatch repair deficient adenoma can vary greatly from $2 \mathrm{~mm}$ upwards. ${ }^{1}$

An important feature present in mismatch repair deficient conventional adenomas from proven mutation carriers was having a villous component. There was also a strong trend for an association between high-grade dysplasia and mismatch repair deficiency. All high-grade adenomas and 93\% of villous adenomas demonstrated mismatch repair deficiency. A previous study of proven mutation carriers (15 individuals with 44 adenomas) also showed this finding. ${ }^{8}$ A further study involving 31 adenomas from 22 mutation carriers found that 15 of 16 high-grade adenomas showed mutation-appropriate loss of mismatch repair, and the remaining adenoma had heterogeneous expression loss. ${ }^{10}$ Taken together, these observations suggest that firstly, high-grade adenomas, irrespective of size or site, represent the most likely lesions in which to screen for Lynch syndrome, and second, that a high-grade lesion that is mismatch repair proficient decreases the likelihood that Lynch syndrome will be diagnosed. Similarly, adenomas with a villous component are also likely to return a result of mismatch repair deficiency in mutation carriers. Patients with Lynch syndrome also developed conventional adenomas which did not show loss of staining. ${ }^{13}$ Notable among these, as would be expected therefore, none had high-grade dysplasia and very few demonstrated a villous component. Though it could be postulated that there are two different populations of conventional adenomas found in mismatch repair mutation carriers, another explanation for these observations is that mismatch repair deficiency accelerates progression. ${ }^{5}$

A previous report has suggested that the gene involved in mismatch repair mutation carriers varies in its capacity to demonstrate loss of expression in conventional adenomas, with MLH1 loss most readily, and MSH6 loss least readily demonstrated. ${ }^{8}$ Our results have also followed this pattern, with over $90 \%$ of adenomas from MLH1 mutation carriers demonstrating loss in contrast to $36 \%$ of $\mathrm{MSH} 6$ mutation carriers. Recent studies have suggested that the Lynch syndrome phenotype is relatively attenuated in MSH6 mutation carriers, ${ }^{23}$ and this may account for a more indolent progression of adenomas in these individuals. In contrast to our findings, another report suggested there was a low detection rate in MLH1 mutation carriers due to heterogeneous staining. ${ }^{12}$ Though heterogeneous staining was present in our series, the number of lesions demonstrating this was very small, and was related to a splicing mutation which may produce a heterogeneous population of mismatch repair proteins. Loss of expression in the majority of adenomas was complete suggesting rapid clonal expansion as a result of mismatch repair expression loss. In two cases, mismatch repair protein loss was not 


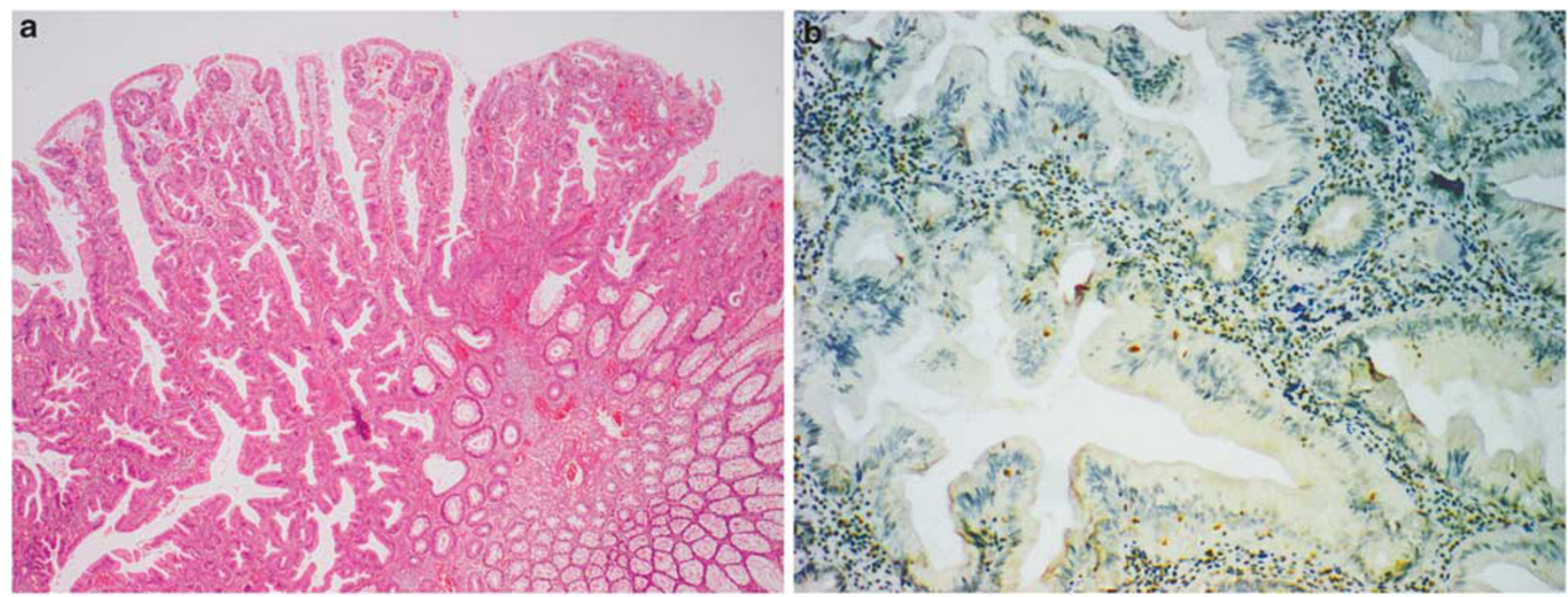

Figure 3 A traditional serrated adenoma in a patient with germline mutation in MLH1 showing loss of expression of the correponding protein in adenoma cells (a: H\&E; b: MLH1 immunohistochemistry).

accompanied by microsatellite instability. Both of these lesions were tubular adenomas, with lowgrade dysplasia; one was $2 \mathrm{~mm}$ and the other $7 \mathrm{~mm}$ in size. The lack of evidence for microsatellite instability, therefore, may be related to a lower proliferative capacity. In a further case, microsatellite instability was present in a tubular adenoma, which showed normal staining of four mismatch repair proteins. This patient carried a missense mutation in $M L H 1$ c. $1865 \mathrm{C}>\mathrm{T}$ p.Pro622Leu and this mutation may have produced a protein with immunoreactivity to an MLH1 antibody. Interestingly, we found that deficiency of mismatch repair proteins was as common in adenomas from the distal colorectum as it was in those from the proximal colon. However, it is well established that colorectal cancers in Lynch syndrome are more consistently found in the proximal colon $^{5,24-26}$ and this suggests that the progression in the proximal colon may be more rapid as has been suggested by other authors. ${ }^{6}$ Other possibilities include differential site exposure to carcinogens in the setting of mismatch repair haploinsufficiency, as well as ease of removal of distal precursor lesions.

In this study, 15 serrated polyps from mismatch repair mutation carriers were also analyzed using immunostaining for mismatch repair deficiency. Loss of expression commensurate with the germline mutation was seen in a subset of these polyps. Interestingly, of five traditional serrated adenomas, four showed appropriate expression loss, and though overall the detection rate using serrated polyps, in general, was decreased when compared with conventional adenomas, the majority of traditional serrated adenomas showed mutation-appropriate loss of expression involving MLH1. Serrated polyps in Lynch syndrome $^{27,28}$ may arise either spontaneously, or in families segregating two genetic predispositions. ${ }^{2}$ In this study, all mismatch repair deficient serrated polyps arose in such a family, ${ }^{2}$ and therefore it is difficult to draw definitive conclusions regarding the usefulness of mismatch repair immunohistochemistry in the diagnosis of Lynch syndrome. There have been previous reports of expression loss in serrated polyps in mismatch repair mutation carriers. Pino et a ${ }^{8}$ reported findings for immunostaining of five hyperplastic polyps from three patients with Lynch syndrome. In two patients with MSH2 mutations, each had a 'pure' hyperplastic polyp where the MSH2 staining had been preserved. In a third patient with an MLH1 mutation, three lesions were described as 'mixed polyps containing elements of hyperplastic polyp and tubular adenoma', with expression loss of MLH1 confined to areas with cytological dysplasia. In this study, we were not able to assess whether other mismatch repair proteins were lost in serrated polyps from mutation carriers due to lack of these lesions from appropriate individuals. Loss of MSH2 expression, for example in a dysplastic serrated polyp, would increase confidence that an individual harbored a Lynch syndrome mutation.

The major strength of this study relates to numbers of cases with known germline mutations. Our study includes 69 proven mutation carriers from the Australasian site of the Colon Cancer Family Registry, ${ }^{14}$ and as such represents the largest series reported so far. Two previous publications examined 44 adenomas from 15 proven mutation carriers ${ }^{8}$ and 31 adenomas from 22 mutation carriers, respectively. ${ }^{10}$ Additional publications have not definitively identified substantive numbers of their subjects as mutation carriers, relying instead on features such as family history of cancer. A limitation of the study is that it does not provide any information on the usefulness of adenoma immunohistochemistry as a screening test for young onset population-based adenomas. A previous study has suggested that an approach using microsatellite instability as a screening test is likely to produce a low yield. ${ }^{9}$ The increasing recognition of the role of family history in determining risk has lead to increased colorectal cancer screening. With increased screening, there will be less colorectal 
cancer available for Lynch syndrome screening. ${ }^{8}$ From our study, we conclude that immunohistochemical mismatch repair testing of adenomas from patients in suspected Lynch syndrome families is a useful alternative in cases where spectrum cancers are unavailable. The overwhelming majority of conventional adenomas from proven mutation carriers showed appropriate loss of mismatch repair proteins, and this was significantly associated with a villous component and a trend to high-grade dysplasia.

\section{Acknowledgements}

This work was supported by grants from the Cancer Council Queensland, the National Health and Medical Research Council, and the National Cancer Institute under RFA CA-95-011 (Australasian Colorectal Cancer Family Registry Centre UO1 CA097735), and through cooperative agreements with members of the Colon Cancer Family Registry and Principal Investigators. The content of this manuscript does not necessarily reflect the views or policies of the National Cancer Institute nor does mention of trade names, commercial products, or organizations imply endorsement by the US Government. JY is a Cancer Council Queensland senior research fellow. CR is a Jass Pathology fellow. The authors also thank the Jeremy Jass Memorial Tissue Bank for supply of polyps for the study.

\section{Disclosure/conflict of interest}

The authors declare no conflict of interest.

\section{References}

1 Iino H, Simms L, Young J, et al. DNA microsatellite instability and mismatch repair protein loss in adenomas presenting in hereditary non-polyposis colorectal cancer. Gut 2000;47:37-42.

2 Walsh MD, Buchanan DD, Walters R, et al. Analysis of families with Lynch syndrome complicated by advanced serrated neoplasia: the importance of pathology review and pedigree analysis. Fam Cancer 2009; 8:313-323.

3 Young J, Simms LA, Biden KG, et al. Features of colorectal cancers with high-level microsatellite instability occurring in familial and sporadic settings: parallel pathways of tumorigenesis. Am J Pathol 2001;159:2107-2116.

4 Lieberman DA, Weiss DG, Harford WV, et al. Five-year colon surveillance after screening colonoscopy. Gastroenterology 2007;133:1077-1085.

5 Jass JR, Stewart SM, Stewart J, et al. Hereditary nonpolyposis colorectal cancer-morphologies, genes and mutations. Mutat Res 1994;310:125-133.

6 Rijcken FE, Hollema H, Kleibeuker JH. Proximal adenomas in hereditary non-polyposis colorectal cancer are prone to rapid malignant transformation. Gut 2002;50:382-386.
7 Pendergrass C. Occurrence of colorectal adenomas in younger adults: an epidemiologic necropsy study. Clin Gastroenterol Hepatol 2008;6:1011-1015.

8 Pino MS, Mino-Kenudson M, Wildemore BM, et al. Deficient DNA mismatch repair is common in Lynch syndrome-associated colorectal adenomas. J Mol Diagn 2009;11:238-247.

9 Ferreira S, Claro I, Lage P, et al. Colorectal adenomas in young patients: microsatellite instability is not a useful marker to detect new cases of Lynch syndrome. Dis Colon Rectum 2008;51:909-915.

10 De Jong AE, Morreau H, Van Puijenbroek M, et al. The role of mismatch repair gene defects in the development of adenomas in patients with HNPCC. Gastroenterology 2004;126:42-48.

11 Muller W, Burgart LJ, Krause-Paulus R, et al. The reliability of immunohistochemistry as a prescreening method for the diagnosis of hereditary nonpolyposis colorectal cancer (HNPCC)-results of an international collaborative study. Fam Cancer 2001;1:87-92.

12 Shia J, Klimstra DS, Nafa K, et al. Value of immunohistochemical detection of DNA mismatch repair proteins in predicting germline mutation in hereditary colorectal neoplasms. Am J Surg Pathol 2005;29: 96-104.

13 Stoffel EM, Syngal S. Adenomas in young patients: what is the optimal evaluation? Am J Gastroenterol 2005;100:1150-1153.

14 Newcomb PA, Baron J, Cotterchio M, et al. Colon Cancer Family Registry: an international resource for studies of the genetic epidemiology of colon cancer. Cancer Epidemiol Biomarkers Prev 2007;16: 2331-2343.

15 Win AK, Dowty JG, English DR, et al. Body mass index in early adulthood and colorectal cancer risk for carriers and non-carriers of germline mutations in DNA mismatch repair genes. Br J Cancer 2011;105: 162-169.

16 Walsh MD, Cummings MC, Buchanan DD, et al. Molecular, pathologic, and clinical features of earlyonset endometrial cancer: identifying presumptive Lynch syndrome patients. Clin Cancer Res 2008;14: 1692-1700.

17 Poynter JN, Siegmund KD, Weisenberger DJ, et al. Molecular characterization of MSI-H colorectal cancer by MLHI promoter methylation, immunohistochemistry, and mismatch repair germline mutation screening. Cancer Epidemiol Biomarkers Prev 2008;17: 3208-3215.

18 Snover D, Ahnen D, Burt R, et al. Serrated polyps of the colon and rectum and serrated polyposis. 4th edn. WHO Classification of Tumours of the Digestive System. IARC: Berlin, 2010.

19 Polydorides AD, Mukherjee B, Gruber SB, et al. Adenoma-infiltrating lymphocytes (AILs) are a potential marker of hereditary nonpolyposis colorectal cancer. Am J Surg Pathol 2008;32:1661-1666.

20 Rijcken FE, Koornstra JJ, van der Sluis T, et al. Early carcinogenic events in HNPCC adenomas: differences with sporadic adenomas. Dig Dis Sci 2008;53: 1660-1668.

21 Ferreira AM, Westers H, Sousa S, et al. Mononucleotide precedes dinucleotide repeat instability during colorectal tumour development in Lynch syndrome patients. J Pathol 2009;219:96-102.

22 Meijer TW, Hoogerbrugge N, Nagengast FM, et al. In Lynch syndrome adenomas, loss of mismatch repair 
proteins is related to an enhanced lymphocytic response. Histopathology 2009;55:414-422.

23 Baglietto L, Lindor NM, Dowty JG, et al. Risks of Lynch Syndrome Cancers for MSH6 Mutation Carriers. J Natl Cancer Inst 2010;102:193-201.

24 Lanspa SJ, Lynch HT, Smyrk TC, et al. Colorectal adenomas in the Lynch syndromes. Results of a colonoscopy screening program. Gastroenterology 1990;98:1117-1122.

25 Vasen HF, den Hartog Jager FC, Menko FH, et al. Screening for hereditary non-polyposis colorectal cancer: a study of 22 kindreds in The Netherlands. Am J Med 1989;86:278-281.
26 Vasen HF, Taal BG, Nagengast FM, et al. Hereditary nonpolyposis colorectal cancer: results of long-term surveillance in 50 families. Eur J Cancer 1995;31A: 1145-1148.

27 Liljegren A, Lindblom A, Rotstein S, et al. Prevalence and incidence of hyperplastic polyps and adenomas in familial colorectal cancer: correlation between the two types of colon polyps. Gut 2003;52:1140-1147.

28 Stoffel EM, Turgeon DK, Stockwell DH, et al. Missed adenomas during colonoscopic surveillance in individuals with Lynch Syndrome (hereditary nonpolyposis colorectal cancer). Cancer Prev Res 2008;1: $470-475$. 